\title{
HAEMATOLOGY IN GENERAL PRACTICE
}

\author{
By D. G. French, M.D.
}

General Practitioner, Ravenswood, Kidsgrove, Staffordshire

\section{Introduction}

The mode of entry into general practice was changed by the introduction of the National Health Service. Formerly it was possible to go straight into practice, even as a principal, as soon as finals were passed, and many did this. The present system ensures that practice vacancies are filled by doctors who have gained some postgraduate experience; many have reached registrar level, and in so doing have acquired competence in some specialty and have been steeped in the scientific method. They may look forward to life in general practice with some dismay, as many imagine that this is so bedevilled by trivialities that no time is left for ' real medicine.' One of the purposes of this paper is to indicate that there is both time and scope for a special interest ... the closer study of some part of one's work to the attainment of an above-average degree of proficiency. It will be learned by experience that there is only time for one special interest at once, though there is no reason why this should not change from time to time. The practical usefulness of haematology in this context is beyond question; so many pathological conditions produce changes in the peripheral blood that a day never passes without some haematological investigation being necessary.

If the work is confined to investigating and treating cases as and when they present themselves, it takes up very little time, but there are so many fascinating questions to be answered that it is only too easy to find oneself undertaking minor research which can be most time-absorbing.

It may be disappointing to find that the interesting cases are rare and that so much of the investigation and treatment of these cases must be handed over to the hospitals, but the major part of the work can be managed completely in general practice and the results are most satisfying. Very little laboratory assistance is needed to confirm the diagnosis of the megaloblastic anaemias, which include pernicious anaemia, megaloblastic anaemia of pregnancy, blind loop syndromes, tropical sprue and non-tropical steatorrhoea, and some post-gastrectomy anaemias; and the same may be said of the leukaemias and aplastic and haemolytic anaemias. Indeed, the investigations required to establish the cause of the commonplace irondeficiency anaemia may need to be more extensive.

\section{Incidence of Anaemia}

It would be an enormous task to establish the total incidence of anaemia in any general practice and I have never attempted to do this. It is possible, however, to give some statistics from this practice of 5,500 patients.

Overwhelmingly the commonest form of anaemia is that due to iron deficiency and most of this is seen in women of child-bearing age. Four years ago I did haemoglobin estimations on a random sample of 500 patients over the age of 50.3 The age was chosen to eliminate anaemia due to physiological repetitive blood loss and the results were most instructive. About $11 \%$ of the total ( 38 women and 19 men) had haemoglobin levels of less than $11.8 \mathrm{~g}$. per $100 \mathrm{ml}$. $(80 \%)$; three were shown to have Addisonian pernicious anaemia, and a fourth, who was complaining at the time of a smooth, painful tongue and whose haemoglobin level was then $14.5 \mathrm{~g}$. per $100 \mathrm{ml}$. (98\%) was also found to have pernicious anaemia about 18 months later; the remainder had iron-deficiency anaemia.

Next in frequency is, therefore, pernicious anaemia, but it is very rare by comparison with iron deficiency. The illusion of frequency is due to the fact that these cases are seen regularly after the diagnosis has been established; they represent just under 3 per 1,000 of this practice population, though it has been shown recently ${ }^{6}$ that the incidence in Great Britain varies in a most interesting way, being lowest in the south-east and increasing fairly regularly to the north. The megaloblastic anaemia of pregnancy is being more frequently diagnosed and this appears to depend to some extent on the skill and persistence applied to the search.

There are four cases of haemophilia in this practice, three of them being brothers with Christmas disease; other uncommon forms of anaemia seen during the past 16 years are shown in Table $\mathbf{I}$. 
TABLE I

Less Common Forms of Anaemia

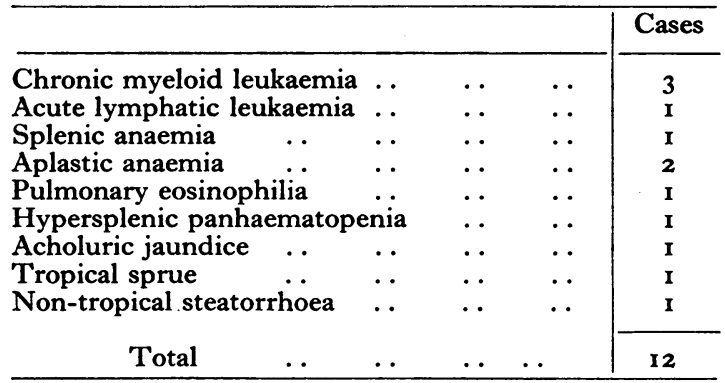

\section{Methods}

When anaemia is suspected, the screen through which all cases must pass is the estimation of the haemoglobin level, so this is a suitable place to discuss the choice of haemoglobinometer. The Sahli instrument is small, compact and cheap; it is capable of producing fairly reliable results when used frequently and carefully ... in fact, that observation applies to all haematological techniques; their dependability varies in proportion to the frequency of their use. The greatest disadvantage of the Sahli instrument is that during the process of colour matching, when the end-point has been passed, the result cannot be checked without cleaning everything and starting again. The most satisfactory instrument for use in general practice is the M.R.C. grey-wedge photometer and its greater expense is fully justified. It is robust in construction and dependable in use; its two great advantages are, firstly that several samples of blood can be taken from different patients and put to one side to be examined at a convenient time within the next two hours; and, secondly, there is no end-point corresponding to that of the Sahli, and the mean of several readings can quickly be determined from the same specimen. Anyone having more than a casual interest in haemoglobin levels should be satisfied with nothing less than this. It gives direct readings in percentages and is calibrated so that $100 \%$ is equivalent to $14.8 \mathrm{~g}$. haemoglobin per $100 \mathrm{ml}$. blood.

If the haemoglobin level is found to be less than I I. $8 \mathrm{~g}$. per $100 \mathrm{ml}$. ( $80 \%$ ) it is necessary to examine the blood more fully and this includes counts of erythrocytes and leukocytes and the preparation of a stained film for an examination of the character and distribution of the cells and for a differential count of 200 leukocytes.

It will be observed that at this point one is left with the colour index as the criterion for differentiating hypochromic, normochromic and hyperchromic anaemias. The alternative to counting erythrocytes is to use a centrifuge to determine the packed cell volume; the mean corpuscular haemoglobin concentration (M.C.H.C.) is then used instead of the colour index and is more reliable. Although erythrocyte counting is laborious and tedious, it is probably quicker than waiting for the centrifuge, and the degree of accuracy of counting depends largely on the care and practice of the operator: I have sometimes regretted not having a haematocrit, but there is much to be said in favour of keeping techniques as simple as possible in general practice; it is better to use simple techniques over the years than to over-complicate the system until it becomes too time-consuming and has to be abandoned in its entirety.

Some practice is required in order to make satisfactory blood films. One should first watch an experienced technician and then practice with one's own blood until some degree of proficiency is acquired. Staining should cause no difficulty but it is important to allow the stain time to act, usually about 17 minutes, and buffer solution at $\mathrm{pH} 6.4$ should be used in place of distilled water. Some modification of the Jenner-Giemsa technique should be adopted for routine use and the finished preparation looks cleaner if stained face-downwards.

Reticulocyte counts must be done in appropriate cases. Mistakes in diagnosis do occur and the failure of treatment to produce a reticulocytosis will probably be the first demonstrable indication of this, when the blood should be re-examined and the diagnosis reconsidered. Cover slips can be prepared at leisure with a saturated solution of brilliant cresyl blue and remain in good condition for months.

Marrow films add enormously to the interest of the work; they are easily made but difficult to interpret. The easiest way to learn the technique is to visit a department of haematology and watch it being done. In general practice one sees too few marrow films for skill to be maintained in their interpretation and it is my custom to make two films, one of which I keep for my own use, while I take the other to a pathologist for an opinion and a demonstration. These occasional visits to laboratories are very profitable and it is unusual to leave without learning something new.

It is useful to know how much time this takes. When a routine has been established, a haemoglobin estimation, erythrocyte and leukocyte counts, preparation of stained film, differential count and replacing the apparatus takes one hour, but need not all be done at one time; using the M.R.C. photometer, the blood may be left for an hour or more before doing the haemoglobin estimation and the film may be left until the next 
day before being stained and examined if the practice is very busy.

Recently a man of sixty came to my consulting room complaining of lassitude, dyspnoea and swelling of the legs and feet. On examination, the only useful finding was a haemoglobin level of $5.9 \mathrm{~g}$. per $100 \mathrm{ml}$. (40\%) and he was sent home to bed. The following day, I called and took blood ${ }^{3}$ for a more complete examination. The evidence now pointed to pernicious anaemia. The next day I called and made marrow films, one of which I took to a pathologist who confirmed the diagnosis. Notice that the patient had been in bed for three days before treatment was started but in that time an accurate diagnosis had been made, and that is an important principle.

\section{Cost of Apparatus}

The cost of apparatus must vary according to the make and model selected but an approximation can be suggested.

A haemacytometer outfit, consisting of counting . chamber, preferably the improved Neubauer, with cover glass, red and white cell pipettes, in case, costs about $£ 4$; a Sahli haemoglobinometer about $£ 3$; M.R.C. grey-wedge photometer $£ 35$; microscope about $f_{5} \circ$; microhaematocrit centrifuge about $£_{65}$. Add to this about $£_{20}$ for stains, flasks and other sundries. Most of this apparatus will give satisfactory service for a lifetime and the cost of expendible items is negligible.

\section{Diagnosis}

The symptoms which cause any anaemic patient to come for advice are numerous and diverse and frequently very vague. Most commonly, they are weakness and tiredness, palpitation and shortness of breath on slight exertion, perhaps accompanied by some swelling of the feet and ankles, especially at night. There may also be angina of effort and it is then easy to allow the diagnosis to drift into the wrong channel.

A patient was once sent from this practice to see a surgeon with a provisional diagnosis of peptic ulcer; the surgeon noticed his dyspnoea, tachycardia and swollen feet and referred him to a cardiologist as a case of heart failure; this diagnosis was confirmed and we eventually received a dissertation on the management of the failing heart. When I saw him later, for the first time, I was impressed by his pallor and investigated his blood in the manner already described, when he was shown to be suffering from pernicious anaemia.

Other presenting symptoms of anaemia include indigestion, dysphagia, persistent cough and nocturnal frequency of micturition; the last of these appears to be due to the fact that fluid collects in the limbs during the day but is returned to the circulation when the patient lies flat in bed and is then excreted.

Pallor of the skin, skin-creases in the palms, mucous membranes and soft palate will usually be evident when the haemoglobin level is less than ro.3 g. per $100 \mathrm{ml} .(70 \%)$ and when iron deficiency anaemia is of long duration there will be koilonychia. The spleen should always be examined; it can be enormous in chronic myeloid leukaemia, hypersplenic panhaematopenia and pulmonary eosinophilia and some palpable enlargement is not uncommon in a variety of other blood diseases.

At this stage the presence of anaemia should be confirmed by a haemoglobin estimation and the investigation must be continued to establish the nature of the anaemia.

If the M.C.H.C. is less than $30 \%$ there is iron deficiency; the haemoglobin level is reduced to a varying degree and the erythrocyte count may be reduced but is frequently within normal limits.

If the M.C.H.C. is greater than $35 \%$, the differential diagnosis includes pernicious anaemia, megaloblastic anaemia of pregnancy, sprue and non-tropical steatorrhoea, acquired haemolytic anaemia, aplastic anaemia and leukaemia; these can only be differentiated by marrow films. It was stressed by Israels ${ }^{4}$ that any patient suspected of suffering from pernicious anaemia should be sent for marrow examination before treatment is given; megaloblasts and normoblasts are quite distinct, but treatment with vitamin $B_{12}$, folic acid or liver causes megaloblasts to change to normoblasts and it is then impossible to establish the diagnosis.

There is a difficult group in which the M.C.H.C. is about $32 \%$. Some of these are due to iron deficiency but many are the result of depressed blood formation associated with other diseases and the prognosis is often very bad. The commoner causes are malignancy, especially of the alimentary tract, the reticuloses and chronic nephritis; I have seen this in cancer of the prostate many months before there was any other indication of the probable source of the primary trouble.

When relying on the M.C.H.C., the commonest trap is the co-incidence of pernicious anaemia with iron-deficiency anaemia and this is most likely to occur in women of child-bearing age. The M.C.H.C. is less than $30 \%$ and the diagnosis in general practice is almost certain to be one of iron-deficiency anaemia. Treatment with iron produces some improvement, as the iron-deficiency element is corrected, but the patient never gets quite well. Re-examination of the blood at this stage reveals a M.C.H.C. of more than $35 \%$. This particular error might be avoided by estimating the mean corpuscular volume (M.C.V.) but any iron deficiency anaemia which has had effective 
treatment and has not been restored to normal in five or six weeks requires reconsideration.

\section{Cause of Iron-Deficiency Anaemia}

Iron-deficiency anaemia can never be regarded as a complete diagnosis and the history must be taken and the patient examined with a view to eliciting the cause. It is necessary to consider tuberculosis at any age, peptic ulcer in young and middle-aged people of both sexes, haemorrhage following parturition, miscarriage or repeated pregnancies and menorrhagia with or without pelvic tumour in women of child-bearing age, and cancer somewhere in men or women over the age of 45. In the last group, other common causes are rheumatoid arthritis, peptic ulcer and piles, though a patient's diagnosis of piles may conceal a carcinoma of the rectum. It is worth remembering, too, that failure to respond to iron may be due to hypothyroidism.

Idiopathic hypochromic anaemia of young males and chronic nutritional hypochromic anaemia of the aged are two dangerous diagnoses which should only be applied in retrospect to cases which have been properly examined and effectively treated, which have remained well after treatment and which in the fullness of time have been shown to have no serious underlying disease.

When an apparently uncomplicated iron-deficiency anaemia is effectively treated, one must be able to demonstrate a reticulocytosis and the subsequent increase in the haemoglobin level must follow a definite pattern. Failure to do so is an indication that the diagnosis is not completely correct, the most probable cause being some complicating factor which has not been found and the aetiology must be reconsidered. One should be perfectly satisfied in every case of iron-deficiency anaemia that the cause has been found.

\section{Treatment of Iron-Deficiency Anaemia}

For oral treatment the iron compounds commonly used are the sulphate, gluconate and succinate and they may be prescribed singly or combined with vitamins and trace elements. Iron is effective when given alone, and if there is some indication for the administration of vitamins they should be prescribed separately, though I have never encountered such a case. My results with the succinate have been so satisfactory that I seldom use anything else, though there is much to be said in favour of a recently produced uncoated tablet which differs considerably in colour, shape and taste from a brand of sweet which is so popular with children, and is therefore some safeguard against accident. Preparations containing cyanocobalamin or folic acid are better avoided altogether.
When a patient responds satisfactorily to oral iron therapy, there is a slight reticulocytosis, first evident about the fourth day and reaching a peak of 5 or $6 \%$ about the eighth day. It is not usually possible to demonstrate an increase in the haemoglobin level until about the seventh to the ninth day and thereafter there is a steady return to normality at the rate of about $1 \%$ per day. The treatment of a fairly severe degree of anaemia takes about two months and may be continued a little longer in the hope of restoring some of the depot iron.

The disadvantage of oral iron therapy is that when the response is poor there must be some doubt about the cause; the patient may not be taking the tablets regularly or the absorption may be poor, especially during pregnancy where I have found the results quite unpredictable; some preparations are not well tolerated, though it is unusual to find a patient who cannot take ferrous succinate. The response to oral iron is slower than to intravenous or intramuscular therapy and this may be important, especially during pregnancy.

Preparations of iron for intravenous or intramuscular administration have a very important place. Those who have an academic interest in anaemia will find that the use of intravenous iron provides a yardstick against which all other forms of treatment can be judged, and they will also find that the hypochromic anaemia of pregnancy is quite incomprehensible without it.

My indications for parenteral iron therapy, which are offered as suggestions, are quite clear-cut and are based on two observations; firstly, that oral iron therapy is unreliable during pregnancy and, secondly, that the results of parenteral iron therapy are so exact and predictable that it has some value in confirming the diagnosis.

So, where the haemoglobin level is less than Ir.8 g. per $100 \mathrm{ml}$. (80\%) I use parenteral therapy in all men, all women over the age of fifty, and all pregnant women. In the remainder I use oral iron therapy and check the progress by means of haemoglobin estimations.

The total dosage of these preparations must be calculated from the patient's weight and haemoglobin level and the results then depend on the interval between the injections; intravenous iron can be given at any time but intramuscular preparations may be painful and it is better not to give the injections more frequently than on alternate days.

A patient having daily injections of intravenous iron will show a significant reticulocytosis on the third day and this usually reaches a peak of about 8 to $10 \%$ on the fifth to the seventh day. Increase in the haemoglobin level can be demonstrated about the fifth to the ninth day after the start of 
treatment and thereafter there is a return to normality at a rate of slightly more than $1 \%$ per day. The first significant increase in the erythrocyte count is observed eight or nine days after the start of treatment and this returns to normal more quickly than the haemoglobin level; in fact there is frequently a period about four weeks after the start of treatment during which counts between five and six million erythrocytes are not uncommon and this is almost certainly due to the fact that the formation of new erythrocytes is taking place at a rate in excess of the destruction and removal of old and effete cells.

\section{The Hypochromic Anaemia of Pregnancy}

The hypochromic anaemia of pregnancy provides a fascinating study for the general practitioner and as a problem it has the enormous attraction of being available at any time. By and large, it is thoroughly badly understood and the present trend of the profession to bury its head in the sand and to prescribe oral iron for the whole duration of the pregnancy leaves much to be desired. One could produce a better argument for giving iron to everyone over the age of fifty.

Experience of ordinary iron-deficiency anaemia cannot be applied directly to the hypochromic anaemia of pregnancy, which is complicated by a physiological hydraemia and gastric achlorhydria. In the known absence of iron deficiency, haemoglobin levels of $10 \mathrm{~g}$. per $100 \mathrm{ml}$. $(75 \%)$ are not uncommon during pregnancy and are uninfluenced by any attempts at curative therapy, and when iron deficiency does exist I have found oral iron therapy unpredictable, certainly after the 2oth week. ${ }^{3}$

Routine haemoglobin estimations have now become part of good antenatal care. If the haemoglobin level is below II.8 g. per $100 \mathrm{ml}$. $(80 \%)$ the blood should be fully examined and, if iron deficiency is demonstrated, the effective treatment is iron by intramuscular injections, the response to which is uninfluenced by the pregnancy and is so rapid that a 'safe' haemoglobin level may be achieved in so short a time as three weeks, though return to complete normality takes longer.

\section{Pernicious Anaemia}

Occasional reference has already been made to pernicious anaemia and as so many of these cases are referred to hospital the whole subject is thoroughly familiar. The diagnosis should be carried as far as possible in general practice and then specialist assistance should be sought to confirm the presence of megaloblasts in the marrow and, whenever possible, for gastric analysis.

Some of these patients present with subacute combined degeneration of the cord, but it can also develop during treatment of the anaemia if adequate dosage of cyanocobalamin is not prescribed $^{1}$ and it may occur in association with any of the megaloblastic anaemias due to vitamin $B_{12}$ deficiency. ${ }^{5}$

On the whole, these patients attend regularly for treatment and their progress can be watched, but a few have their injections given by district nurses and must be visited three or four times a year to ensure that adequate blood levels are being maintained.

\section{Uncommon Forms of Anaemia}

Other forms of anaemia are so rare in the experience of a general practitioner that they have no place in a paper of this kind. When they do occur, much can be learned by taking the diagnosis as far as possible by these simple means, but in the end further aid must be sought, as the final diagnosis and treatment require hospital facilities. Even so, they are responsible for a large part of that stimulus which is so necessary for the maintenance of interest.

\section{Conclusion}

In conclusion, it must be emphasized that every practice has a surprising number of anaemic patients who struggle along in subnormal health quite needlessly and whose condition cannot be diagnosed by looking at the lips and conjunctivae. The only means of detecting anaemia is by the use of the haemoglobinometer, and having found it the search must be continued for malignant disease, hypothyroidism and the various other conditions already mentioned. There is no other specialty in general practice which is so far reaching as haematology.

\section{REFERENCES}

1. ADAMS, J. F., and TIMBURY, G. C. (1959), Brit. med. F., i, 883.

2. FRENCH, D. G., 'British Encyclopaedia of Medical Practice,' Interim Supplement, 190, London, p. 2.

3. FRENCH, D. G. (1955), Proc. roy. Soc. Med., 48, 347.

4. ISRAEIS, M. C. G. (1955), Ibid., 48, 353.

5. NICOL, W. A. (1960), Brit. med. F., i, 322.

6. SCOTT, E. (1960), f. Coll. Gen. Pract., 3, 80. 